\title{
Ill-Defined Knowledge Boundaries; A Battlefield for Transdisciplinary Engineering
}

\author{
Nikki Kaasgaard JEPSEN ${ }^{1}$ and John Bang MATHIASEN \\ Aarhus University, Department of Business Development and \\ Technology, Denmark
}

\begin{abstract}
Relying on an one-year longitudinal study of developing a product to be assembled in refugee camps around the globe, this paper aims at studying whether a battlefield exists among the practitioners involved, caused by ill-defined knowledge boundaries and how to penetrate these. The paper juxtaposes the empirical material with the theoretical conceptualisation of affordance to show that the drawbacks of knowledge boundaries emerged after long and intense discussions rather late in the development. Indeed, it was necessary to arrange a workshop in which the practitioners gained hands-on experience to reveal the drawbacks of knowledge boundaries. To mitigate drawbacks of knowledge boundaries, this paper suggests that transdisciplinary engineering should be a fundamental activity, which takes place throughout the development. Practitioners facing ill-defined knowledge boundaries should adapt and apply a common framework including various artefacts to guide both the practical work and their discussions to gain hands-on experience.
\end{abstract}

Keywords. Transdisciplinary Engineering, Manufacturability, Affordance, Knowledge Boundary

\section{Introduction}

The practical reality of developing new products reveals that knowledge boundaries between different professional disciplines exist [1]. The knowledge boundaries result in some practitioners either lack understanding of manufacturability [2;3] or that they focus on functionality at the expense of manufacturability [4]. Likewise, a study of product development focusing on prototype- and mainstream manufacturing issues [5] shows knowledge boundaries between different professional disciplines, which leads to different perceptions of functionality and manufacturability. These knowledge boundaries cause an ill-defined problem where no obvious solution exist.

Transdisciplinary engineering is a useful approach to handle ill-defined problems, such as knowledge boundaries [6] given that it enables different professional disciplines to achieve a balance between functionality and manufacturability. However, given that each group of professional disciplines often operates within different practices [7] and the fact that they speak different language [8], the applied knowledge is embedded in a practice in which both social and technical matters influence the possibilities for action 
[9] and knowledge is continuously modified [10]. This embeddedness and temporal nature of knowledge challenges transdisciplinary engineering [1] because sketches, drawings and prototypes offer different possibilities for actions for the involved practitioners. These different possibilities for actions can lead to points of contentions between the involved practitioners and the result of these may very well be that a battlefield is created between the different professional disciplines.

To the best of the authors' knowledge, researchers have paid little attention to the practical realities of ensuring balance between functionality and manufacturability, and more importantly, we lack understanding of how to realise that a battlefield exists and how to handle the battlefield between the involved transdisciplinary practitioners. The battlefield is the consequence of unmanageable knowledge boundaries, but given that knowledge is temporal, the knowledge boundary does also have a temporal nature. Accordingly, by focusing on how knowledge boundaries unfold when doing transdisciplinary engineering, the purpose of this paper is to expose the battlefield and thus study how to penetrate these knowledge boundaries. The research questions guiding the study are: what influence do knowledge boundaries have on transdisciplinary engineering? and how to mitigate the consequences of knowledge boundaries?

The theoretical conceptualization of our study combines the concept of affordance [11], possibilities for actions in a sociotechnical practice with managerial approach to facilitate balance between functionality and manufacturability [4].

The empirical settings for this research are the development and prototype manufacturing of a product in a high-wages area, while the final assembly should be done in different refugee camps around the globe. This paper explores empirically the process of balancing functionality and manufacturability after the first 100 products were assembled over a period of two and a half months. One of the authors draws on Moultrie and Maier's [4] simplified model to study the process of balancing functionality and manufacturability matters.

In the following, we present the theoretical positioning, followed by methodological considerations and the presentation of the case. Next, we analyse the case and present the discussion and conclusion of the paper.

\section{Theory}

Following the background for this study, we present the applied conceptualisation of affordance and the framework for accomplishing the analysis.

\subsection{Background for the study}

The concept of manufacturability and design for assembly were introduced in the 1960s [12]. By taking design for manufacturing and design for assembly into consideration during the design phases, it is possible to reduce the overall cost of the product [13]. Yet, the design phases of product development often have a focus on functionality and this focus is strengthened by the physical distance from the designer of the manufacturing [4]. This happens even though balancing functionality and manufacturability have achieved huge attention since Boothroyd and Dewhurst introduced the Concurrent Engineering (CE) concept in the early 1980s.

A generic model to ensure a structured and coordinated CE is the "stage/gate" approach e.g. [14]. Based on this viewpoint, it is advised to postpone decisions until 
sufficient knowledge of the issue to be handled has been achieved. This way of managing the development is the focal point for Sanders and Klein's [15] "integrated productindustrial" approach. Their approach highlights a need for coordinating the development in terms of the "voice of the customers" with the "voice of manufacturing" at different levels of the product; i.e., system-, sub-system-, module- and component level; please see Hsuan [16] for an elaboration of these four levels. Basically, the "integrated productindustrial" approach focuses on balancing users' needs with manufacturability.

To gain an understanding of both the "voice of the customers" and the "voice of manufacturing", the practitioners have to cross knowledge boundaries $[17 ; 18]$. However, throughout the processes of $\mathrm{CE}$ new requirements might emerge, prioritisation might change, and the achieved knowledge might reveal that past decisions are now inappropriate. Likewise, transdisciplinary engineering involves individual practitioners from various professional disciplines, who operate within different practices and thus make use of different terminologies. This transdisciplinary nature of doing CE combined with the mutual requirements will form knowledge boundaries. Carlile [19] exemplifies the consequences of knowledge boundaries in his study of product development; "In this design review meeting, Mick's statements about the "awkwardness of the design" only seemed to be inflaming the tempers of the sales representative and design engineers on the team.". Apparently, the knowledge boundaries between transdisciplinary engineers created a battlefield of opinions.

Knowledge boundaries influences the practitioners' opportunities for action. Indeed, transdisciplinary engineering entails, that the possibilities for actions differ across knowledge boundaries. With the aim of studying the different possibilities for action, we draw on the concept of affordance.

\subsection{On the term Affordance}

After Gibson [20] published his famous book "Ecological Approach to Visual Perception", the concept of affordance has been applied across multiple research domains to study the reciprocity between human (social) and artefacts (technical). In 1988, affordance was introduced to the design domain by Norman [21] with his famous book "the psychology of everyday thing". Norman's conceptualisation of perceived affordances is different from Gibson's as Norman highlights that affordance depends on a human's embodied knowledge. In other words, affordance emerges in the humanartefact reciprocity.

The conceptualisation of affordance in this paper is slightly different: Firstly, a practitioner's thinking and action is inseparable and situational; it is a "thinking-inaction" process [22]. This process is influenced by artefacts [9] and the social interaction with other practitioners [8]. This means that the technical matters (artefacts) and social matters (social interactions) acquire meaning for the individual practitioner and in this way gain an influencing role on the practitioner's actions when handling a problematic situation. In other words, affordance is enacted when a practitioner(s) gradually handles a problematic situation by conducting a "controlled transformation of an indeterminate situation into a determinately unified one" [22]. Secondly, practitioners have different faculties [22] and they also have different commitment for acting [23] and given that applied language differs $[8 ; 24]$ different artefacts can facilitate different understandings, when practitioners handle an indeterminacy. In other words, affordance will differ among the involved practitioners. Thirdly, drawing on inspiration from the work of Carlile [1] and Leonardi [25], three types of affordance is conceptualised; (1) shared, (2) 
collective, (3) pragmatic. Shared affordance has the underlying assumption that all practitioners have the same possibilities for actions. Collective affordance differs because there are now different possibilities for actions between the practitioners involved and finally pragmatic affordance means that the possibilities for actions are situational and therefore embedded in a given problematic situation. Fourthly, when accomplishing transdisciplinary engineering the cues from the customers (i.e. functionality requirements) are converted into product knowledge and manufacturing knowledge [26]. When enacting this process, artefacts as for instance diagrams and sketches facilitate practitioners to convert the cues from customers into applicable knowledge. These artefacts are however merely 'stand-ins' for the practitioners' object of design, and later they emerge as the physical product [27]. This means that the different cues that invites a practitioner to act upon a given artefact are temporal by nature. In the following, we present the framework for accomplishing the analysis.

\subsection{The framework for the analysis}

A method to cross knowledge boundaries, and thusly mitigate the effect of their existence, is Appelton and Garside's [28] team-based approach to design for assembly. Yet, in later studies this team-based approach was found to be too complex and time consuming in many situations, e.g. [4]. Accordingly, Moultrie and Maier [4] developed "the simplified approach", which can help to reveal the practitioners' knowledge of manufacturability. The simplified model consists of six steps; Firstly, the baseline metrics has to be established to ensure a common goal and thus focus for the transdisciplinary team. Secondly, the product has to be assessed on a system level before it is disassembled and placed in an exploded view to expose subassemblies. Thirdly, the essential parts of the product is to be determined by assessing the strength of the function(s) of that part. To aid this process all parts are to be given a name with a card placed next to the part. Fourthly, the product is reassembled while process cards are created for each step and each part is scored in terms of how difficult it is to handle, fit or fix. The process and part cards are placed as a manufacturing flow. Fifthly, the product, parts and processes are critiqued and ideas for improvements are generated based on the critique. Sixthly, the product is redesigned with the focus of achieving the baseline metrics established in the preliminary phase.

Given that the aim of the simplified approach is to facilitate a transdisciplinary team consisting of $4-6$ practitioners to put a laser like focus on both the "voice of the customers" and the "voice of manufacturing" this research draws on this approach to study knowledge boundaries as far more aspect will come into play [29].

\section{Methodological considerations}

This research is based on a one-year longitudinal case study of a company that has developed a product and started the manufacturing of the first generation of the product. The intention of the company is to draw on the knowledge gained from producing 100 products to do minor design changes to enable the manufacturing to be moved from the current high wage settings to local manufacturing in the refugee camps. We use this case to empirical provide insight and thus improve the understanding of how social and technical matters influence the knowledge boundaries and how to handle these illdefined knowledge boundaries. 
The analysis in this paper is an iterative back and-forth approach that switches between theory and empirical data. This abductive approach [30] is useful because there was a need for ongoing back and-forth between the collected empirical data and our theoretical conceptualisation.

The data collection consists of observations done during the product development phases in the winter and spring of 2018, the subsequently assembly process of the first 100 units in the fall of 2018 and finally observations during a workshop where the responsible salesman and all Product Development Engineers' (PDE) and Industrial Engineers' (IE) attended with the goal of improving the manufacturability by utilizing Moultrie and Maier's [4] simplified model.

\section{Case}

To make the empirical data more reader-friendly the case is written in chronological order. Before presenting the case, as a matter of form it should be mentioned that the PDEs and the IEs have received teaching in the subject of manufacturability and functionality from the same theoretical curriculum in consecutive years at the same university. The salesman has not received official training in the subject, but has worked extensively alongside the aforementioned PDEs.

In January 2018, the product moved from a concept into the hands of the PDEs. The required functionality of the product was clarified by using three sources of information; firstly, first hands experience gained by the salesman and the PDEs by visiting a refugee camp. Secondly, an end-user questionnaire consisting of information from 44 respondents and thirdly, information from UN's 17 goals for sustainable development. The PDEs used these functional specifications to create all specifications for the product. Following an approval of these product specifications, the PDEs translated these specifications into a physical product design; i.e., the specifications enabled the PDEs to fulfil the required functionality. One of the essential requirements was that the product had to be sustainable; the company perceives sustainability as the ability to offer local assembly jobs (in the refugee camps) during the manufacturing process.

In the fall of 2018, a working physical prototype was produced, and 100 units were sold. Subsequently, the product moved from the hands of the PDEs to the hands of the IEs. To keep the record straight, both the salesman, PDEs and IEs were actively involved in the manufacturing process of the 100 products. The manufacturing process took place over a period of two and a half month. During this time, several conflicts arouse about the design of product, for instance; (1) all wires were attached by soldering and the PDEs deemed this to be a viable solution, while the IEs did not believe that the intended worker located in refugee camps around the globe would be able to complete such a manufacturing task: (2) several connection points needed three or more wires connected by soldering - the IEs believed this to be almost impossible to perform, but the PDEs thought it would not be problematic since they could do it: (3) many parts were mounted from awkward angles making the assembly process very difficult - this was a big concern for the IEs, but once again the PDEs believed this to be a silly concern, since it was only a matter of repositioning yourself to the relevant task.

After completing the manufacturing of the 100 products, the IEs were supposed to make a few small changes to the product's design that would enable a manufacturing of at least 5000 products within a reasonable time-frame. This was seen by the IEs to be an impossible task because they thought the architecture of the product was immature and 
needed significant development. Neither the PDEs nor the salesman did understand the IEs' concerns regarding the problematic product architecture. It is worth remembering that the intended workers, who should perform the final manufacturing in the refugee camps, are low skilled; the intention that people living in the refugee camps should be capable of assembling the product had already led to several conflicts.

To achieve the goal of manufacturability in the refugee camps and to share knowledge between the involved employees, the salesman and all PDEs and IEs involved were invited to a workshop on this subject. To structure the workshop Moultrie and Maier [4] simplified six-steps model for design for assembly was applied. In the workshop the following issues were observed during each of the six steps; Step 1) baseline metric of manufacturability was established and agreed upon. The manufacturability had to be assessed from the assumed low skill level of a refugee living in the camp. Step 2) the product was analysed and discussed on a system level. To guide the discussion the scorecard design by Moultrie and Maier [4] was used. The participants used the physical product to explain what they meant. This helped both the IEs, and the PDEs to elaborate different problematic issues, e.g. "what is an awkward work angle" and exemplify what they meant. The disassembly process revealed that several parts could not be removed without damaging the product. Step 3) essential parts were identified after agreeing on how to define essentiality. Each component was given a name to support a clarification of the function of the part. Step 4) during this step it became evident that the product needed a fundamental redesign. When scoring all the different parts in "handling", "fitting" and "fixing" the salesman/PDEs/IEs team gradually gained a common understanding that the current assembly process was far too complex. This led to several rather intensive discussions on what could be done to fix these problems; however, this was not the intention of the fourth step according to the Moultrie and Maier [4] approach. The reassembly process was not completed to a satisfactory level; apparently, the motivation for finalising step 4 gradually disappeared into thin air, mainly due to the realisation that the product needed to be completely redesigned. Step 5) and Step 6) were combined and it was mainly a continuation of the previously step 4 . In other words, the salesman, PDEs and IEs picked up the intensive discussions from where these ended. During these discussions for improvement, several artefacts were applied to explain the different ideas, e.g. drawing, power cords, computer frames, power stations, pictures of car chassis and more. Apparently, both the PDEs and salesman acknowledge that the current design of the product resulted in an awkward manufacturing process. Three of the many realisation-statements the salesman and PDEs put forward were; (1) "why not consider some self-locking mechanism for the different plugs", (2) "we need interfaces between the different elements that only fits in one place. In this way it would be fool proof to assemble and not like it is at the moment where any wire can be soldered on any pin" and (3) "actually, do we need to develop a platform on which we can build and develop the product?".

\section{Analysis}

In an iterative manner, we juxtapose our conceptualization of affordance presented in the theoretical section with the one-year longitudinal case in the preceding section to answer, what influence do knowledge boundaries have on transdisciplinary engineering? and how to mitigate the consequences of knowledge boundaries? 
As explained in the case the two ground of engineers involved have equal educational background in terms of balancing functionality and manufacturability and the salesman have practical experience gained from working with the engineers. Thus, it could have been opportune to assume that a shared affordance existed between the involved salesman, PDEs and IEs since they all things being equal shared common knowledge with regards to the concepts of manufacturability and functionality. This would have provided the involved with the same possibilities for action during the redesign of the product, and thusly have reduced the likelihood for forming the battlefield(s).

As illustrated in the case, when the task of developing the product was handed over to the PDEs, they started with examining the functional requirements derived from three different sources; visiting a refugee camp, the questionary and UN's 17 goals for sustainable development. In other words, the cues from these three sources afforded the PDEs to draw up all functional, product and manufacturing specifications. Despite an essential manufacturability requirement was to achieve a sufficient level of manufacturability that would enable local manufacturing in the refugee camps, the PDEs developed and produced a physical prototype that according to their knowledge fulfilled all requirement. However, the PDEs completed the product development process from concept to prototype without involving the IEs and the cues from the different source did not afford a design fulfilling both functionality and manufacturability requirements.

The assembly process led to several conflicts between the salesman, PDEs and the IEs. All the discussions were about the manufacturability of the product. The cues from the product/manufacturing specifications afforded the PDEs to assemble the product, but it did not afford the IEs to assemble the product in a proper way. In addition, the IEs emphasised several times that due to the refugees do not possess sufficient knowledge, the cues from the manufacturing specifications will not afford manufacturing in the refugee camps. Thus, due to knowledge boundaries between the salesman, PDEs, IEs and refugees the cues from the specifications resulted in four different kinds of individual affordances. In the attempt to mitigate these ill-defined knowledge boundaries and thus achieve shared affordance, several discussions took place. Only the produced prototype was applied to guide and structure the discussions. On the one hand, the PDEs suggested modifying the manufacturing set-up in the refugee camps, which resulted in a battle addressing the understanding of the term manufacturability. On the other hand, the IEs was sure that the problem was not because of the manufacturing set-up, but the real problem was the architecture of the product, which they believed to be immature. Given that these long-lived and fierce discussions did not result in any changes of the product or manufacturing set-up the knowledge boundaries remained ill-defined.

After handing over the improvement task to the IEs, which should enable mainstream manufacturing in the refugee camps, the consequences of the ill-defined knowledge boundaries gradually became evident. Obviously, the product needed to have a better balance between manufacturability and functionality. However, it was neither the ongoing discussions nor the use of sketches, drawings and model when producing the first 100 products, which paved the way for acknowledging the ill-defined knowledge boundaries. Rather, it was the practical application of Moultrie and Maier's [4] simplified model during the one-day workshop process, which afforded the salesman, PDEs and IEs to acknowledge and subsequently gain a sufficient understanding of the knowledge boundaries and thus the consequences of their individual affordances. At the workshop, the salesman, the PDEs and IEs followed the simplified model rigorously in the first half of the session. The model afforded the practitioners to bring manufacturing issues at the refugee camps to the fore and to agree on the scoring of manufacturability issues. 
However, it was the discussions during the fourth step in which the consequence of the ill-defined knowledge boundaries burst into flames. The exploded view of the disassemble product combined with drawings, sketches, online pictures, laptop, a disassemble computer and power supply devices which made it possible for the salesman, PDEs and IEs to penetrate the knowledge boundaries. This led to the realization that the product needed a completed redesign, and that is was crucial to involve several functions; the salesman, the PDEs and the IEs.

\section{Discussion}

The findings in this study show the consequences of bypassing transdisciplinary engineering from the outset of developing new products. The mainstream manufacturing will take place in refugee camps in which the both the social and technical matters are completely different from the contextual settings where the product has been designed and the prototype manufacturing has been accomplished. These social and technical differences were ignored, which implies that the ill-defined knowledge boundaries between the different groups of practitioners were not an issue during the design and prototype manufacturing. It resulted in the consequences of the ill-defined knowledge boundaries were subdued until the product was handed over from prototype to mainstream manufacturing. These findings have some similarities to Jalonen et al.'s [5] account of the consequences of having two coexisting prototype- and mainstream manufacturing; yet, in our study we show that the real source of the problem is the lack of transdisciplinary way of handling ill-defined problems throughout the development. For instance, the PDEs had a tendency to unwittingly ignore "the voice of the manufacturing" (the social and technical circumstances in the refugee camps) and thus unconsciously postponed the conflict with the IEs. One of the sources of conflict was the balance between manufacturability and functionality; particularly, the term "manufacturability" was perceived differently by the different practitioners. In the case, we witness that the conflict aroused in the manufacturing at the time of involving the IEs, leading to several heated debates about manufacturability. Even though there were several discussions, none of them resulted in changes of the product since the IEs were incapable of convincing the PDEs that a radical change of the product was a pivotal prerequisite for manufacturing the product in a refugee camp. Accordingly, if facing illdefined knowledge boundaries it is paramount that the practitioners apply a transdisciplinary approach to gain sufficient understanding of both social and technical matters.

Doing transdisciplinary engineering entails that practitioners have different faculties [22] and commitment for acting [23], which influences the perception of the knowledge boundary; for instance the PDEs, IEs and the salesman acted rather differently during the development phases and thus faced different knowledge boundaries. Likewise, by drawing on the concept of affordance - possibilities for actions - the analysis illustrates that the knowledge boundaries are also influenced by the different practitioners' perception of the transdisciplinary problem to be handled. Obviously, the transdisciplinary problem and thus ill-defined knowledge boundaries existed among the involved practitioners at the outset of the development, but the ill-defined knowledge boundaries did not have any influence on the development before the salesman, PDEs and IEs jointly revealed the ill-defined problem during the workshop. The knowledge boundaries were ill-defined until the workshop and as Carlile [1] reminds us it can be 
rather resource demanding to penetrate knowledge boundaries; to share knowledge across boundaries calls for either a transfer, translate or transform approach. This paper subscribes to the findings of Carlile [1], but our study shows that in some situation the representation capacity of only a boundary object is insufficient to penetrate ill-defined knowledge boundaries. For instance, to penetrate the knowledge boundaries it was necessary to give the PDEs, salesman and IEs a common hands-on experience, which also required the use of an exploded view of the physical disassemble product combined with drawings, sketches, online pictures, laptop, a disassemble computer and power supply devices.

If facing ill-defined knowledge boundaries when doing transdisciplinary engineering this study suggest conducting a workshop in which the practitioners involved apply a model as for instance Moultrie and Maier's [4] to gain a common hands-on experience. Having a common framework to guide both the discussions and practical work combined with the use of various artefacts, practitioners will be capable of overcome the ill-defined knowledge boundaries.

One could argue that if a good balance between manufacturability and functionality is desired the solution is simple to allow the IEs to do the product development. However, this is not a viable solution since they have a tendency to ignore "the voice of the customer", thus creating an unbalance between functionality and manufacturability by favouring the side of manufacturability. Hence, the analysis illustrates that the battle between practitioners involved should not take place during the manufacturing stage, but if a battlefield is necessary this paper suggests moving the essential clarification of achieving an appropriate balance between manufacturability and functionality far upstream the development process.

\section{Conclusion}

At the outset, the purpose of this paper was to expose whether a battlefield exists and thus study how to penetrate these knowledge boundaries. The research draws on a oneyear longitudinal case of developing a product to be manufactured by refugees on location in the camp.

The influencing roles of knowledge boundaries were not realised during the design thus enabling the functionalities of the product at the expense of manufacturability. The drawbacks of the ill-defined knowledge boundaries burst into flame when the IEs arranged a workshop with the purpose of illustrating the lack of manufacturability in the current design. To mitigate the consequences of the ill-defined knowledge boundaries this study suggests that:

- Companies doing transdisciplinary engineering should take into consideration whether an individual, shared or collective affordance exists among the practitioners

- The source of problematic and ill-defined knowledge boundaries is a lack of real transdisciplinarity throughout the product development. Thus, transdisciplinary engineering should be a fundamental activity taking place throughout the development

- Companies doing transdisciplinary engineering should not avoid the orchestration of a battlefield between different professional disciplines. Doing so will only postpone the problem to more downstream stages. 
- Companies facing ill-defined knowledge boundaries could arrange a workshop to gain a common hands-on experience. In such situation a common framework to guide both the discussions and practical work combined with the use of various artefacts practitioners enables engineers to penetrate knowledge boundaries

\section{References}

[1] P.R. Carlile, Transferring, translating, and transforming: An integrative framework for managing knowledge across boundaries. Organization science, 2004, 15(5), pp. 555-568.

[2] C. Poli, P. Dastidar and R. Graves Design knowledge acquisition for DFM methodologies. Research Engineering Design, 1992, Vol. 4, pp. 31-145.

[3] C.L. Tan and M. Tracey, Collaborative new product development environments: Implications for supply chain management. Journal of Supply Chain Management, 2007, 43(3), pp. 2-15.

[4] J. Moultrie and A. Maier, A simplified approach to design for assembly. J Eng Des, 2014,25(1-3), 44-63.

[5] M. Jalonen, Ristimäki, P., Toiviainen, H., Pulkkis A., \& Lohtander, M., Between product development and mass production, Journal of Workplace Learning, Vol. 28:1, 2016, pp.33-48

[6] Wognum, N., Bil, C., Elgh, F., Peruzzini, M., Stjepandić, J., \& Verhagen, W. J. (2019). Transdisciplinary systems engineering: implications, challenges and research agenda. International Journal of Agile Systems and Management, 12(1), 58-89.

[7] Jałowiecki, A., Kłusek, P., \& Skarka, W. (2017). The Methods of Knowledge Acquisition in the Product Lifecycle for a Generative Model's Creation Process. Procedia Manufacturing, 11, 2219-2226.

[8] L.L. Bucciarelli, Between thought and object in engineering design. Design Stud, 2002, 23(3), $219-231$.

[9] K. Henderson, On Line and on Paper: Visual Representations, Visual Culture, and Computer Graphics in Design Engineering, MIT Press, Cambridge, 1999.

[10] A. Zouari, M. Tollenaere, H.B. Bacha and A.Y. Maalej, Domain knowledge versioning and aggregation mechanisms in product design, Conc Eng: Research and Applications, Vol. 23:4, 2015, pp. 296 - 307.

[11] L. van Dijk and E. Rietveld, Foregrounding sociomaterial practice in our understanding of affordances: The skilled intentionality framework. Frontiers in psychology, 2017, 7, 1969.

[12] J.G. Bralla, Design for manufacturability handbook (Vol. 11), McGraw-Hill, New York, 1999.

[13] K.T. Ulrich and S.D. Eppinger, Product Design and Development. McGraw-Hill, New York, 2000.

[14] K.T. Ulrich and S.D. Eppinger, Product Design and Development, McGraw-Hill, New York, 2016.

[15] A. Sanders and J. Klein, Systems Engineering Framework for Integrated Product and Industrial Design Including Trade Study Optimization, Procedia Computer Science, Vol. 8, 2012, pp. 413-419.

[16] J. Hsuan, Impacts of supplier-buyer relationships on modularization in new product development. European Journal of Purchasing \& Supply Management, 1999, 5(3-4), pp. 197-209.

[17] Star, S. L., \& Griesemer, J. (1999). Institutional ecology, translation and boundary objects. The science studies reader, pp. 505-524.

[18] B. Ewenstein and J. Whyte, Knowledge practices in design: the role of visual representations asepistemic objects'. Organization studies, 2009, 30(1), 07-30.

[19] P.R. Carlile, A pragmatic view of knowledge and boundaries: Boundary objects in new product development. Organization science, 2002, 13(4), 442-455.

[20] J.J. Gibson, The ecological approach to visual perception. Routledge, Boston, 1979.

[21] D.A. Norman, The psychology of everyday things (Vol. 5). Basic books, New York, 1988.

[22] J. Dewey, Logic: The Theory of Inquiry, Southern Illinois University Press, Carbondale, 1938.

[23] B. Elkjaer, Organizational learning: the 'third way'. Management learning, 2004, 35(4), 419-434.

[24] M. Mottonen, J. Harkonen, P. Belt and H. Haapasalo, Managerial view on design for manufacturing, Industrial Management \& Data Systems, Vol. 109:6, 2009, pp. 859-872.

[25] P.M. Leonardi, When does technology use enable network change in organizations? A comparative study of feature use and shared affordances. MIS Quarterly, 2013, 749-775.

[26] M. Borsato and M. Peruzzini, Collaborative Engineering, In: J. Stjepandić et al. (eds): Concurrent Engineering in the 21st century. Foundations and Challenges, Springer International Publishing Switzerland, 2015, pp. 165-196.

[27] L.L. Bucciarelli, An ethnographic perspective on engineering design. Design Stud, 1988, 9(3), 159-168.

[28] E. Appleton and J.A. Garside, A team-based design for assembly methodology. Assembly Automation, 2000, 20(2), 162-170.

[29] D.E. Whitney, Manufacturing by design. Harvard Business Review, 1988, 66(4), pp. 83-91.

[30] A. Dubois and L.E. Gadde, Systematic combining: an abductive approach to case research. Journal of Business Research, 2002, 55(7), 553-560. 